\title{
Similarity Solutions for Flow and Heat Transfer of Non-Newtonian Fluid over a Stretching Surface
}

\author{
Atta Sojoudi, ${ }^{1}$ Ali Mazloomi, ${ }^{2}$ Suvash C. Saha, ${ }^{3}$ and Y. T. Gu ${ }^{3}$ \\ ${ }^{1}$ Department of Mechanical Engineering, College of Engineering, University of Tehran, P.O. Box 11155-4563, Tehran, Iran \\ ${ }^{2}$ School of Mechanical Engineering, Sharif University of Technology, P.O. Box 11155-8639, Tehran, Iran \\ ${ }^{3}$ School of Chemistry, Physics and Mechanical Engineering, Queensland University of Technology, 2 George Street, \\ GPO Box 2434, Brisbane, QLD 4001, Australia
}

Correspondence should be addressed to Suvash C. Saha; s_c_saha@yahoo.com

Received 19 January 2014; Revised 24 April 2014; Accepted 24 April 2014; Published 11 May 2014

Academic Editor: Subhas Abel

Copyright (C) 2014 Atta Sojoudi et al. This is an open access article distributed under the Creative Commons Attribution License, which permits unrestricted use, distribution, and reproduction in any medium, provided the original work is properly cited.

\begin{abstract}
Similarity solutions are carried out for flow of power law non-Newtonian fluid film on unsteady stretching surface subjected to constant heat flux. Free convection heat transfer induces thermal boundary layer within a semi-infinite layer of Boussinesq fluid. The nonlinear coupled partial differential equations (PDE) governing the flow and the boundary conditions are converted to a system of ordinary differential equations (ODE) using two-parameter groups. This technique reduces the number of independent variables by two, and finally the obtained ordinary differential equations are solved numerically for the temperature and velocity using the shooting method. The thermal and velocity boundary layers are studied by the means of Prandtl number and non-Newtonian power index plotted in curves.
\end{abstract}

\section{Introduction}

Non-Newtonian fluids such as foodstuffs, pulps, glues, ink, polymers, molten plastics, or slurries are increasingly used in various manufacturing, industrial, and engineering applications, particularly in the chemical engineering processes. Many simultaneous transport processes exist in industrial or engineering applications. For instance, mechanical forming processes include extrusion and melt spinning where the extruded material issues through a die. The ambient fluid status is stagnant but a flow is induced adjacent to the material being extruded, due to the moving surface. Noting that the fluids employed in material processing or protective coatings are in general non-Newtonian, so the study of nonNewtonian liquid films is important.

The problem of heat and mass transfer from vertical plate has been the subject of great interest for several researchers in past decades. As an earlier study, Wang [1] considered the flow problem within a finite liquid film of Newtonian fluid over an unsteady stretching sheet. Later, Andersson et al. [2] investigated the heat transfer characteristics of the hydrodynamic problem solved by Wang [1]. Usha and Sridharan [3] regarded a similar problem of axisymmetric flow in a liquid film. The effect of thermocapillarity on the flow and heat transfer in a thin liquid film was studied by Dandapat et al. [4]. The free-surface flow of non-Newtonian liquids in thin films is a widely occurring phenomenon in various industrial applications, for instance, in polymer and plastic fabrication, food processing, and coating equipment. There are limited papers on gravity-driven power-law film flows [5-7] and the studies of non-Newtonian film flows on an unsteady stretching surface remain little. The heat transfer aspect of such problem has also been considered by Chen [8].

The most distinctive works of Vajravelu and Hadjinicolaou [9] and Mehmood and Ali [10] are available in the literature describing the heat transfer characteristics in the 
laminar boundary layer of a viscous fluid over a linearly stretching continuous surface with variable wall temperature and the incompressible generalized three-dimensional viscous flow with heat transfer analysis in the presence of viscous dissipation generated due to uniform stretching of the plane wall, respectively. Unsteadiness of the similar problems is stated by many researchers. For instance, Tsai et al. [11] studied nonuniform heat source/sink effect on the flow and heat transfer from an unsteady stretching sheet through a quiescent fluid medium extending to infinity. The boundary layer equations are converted by using similarity analysis to be a set of ordinary differential equations including unsteadiness parameter.

Recently, Sahoo and Do [12] investigated the entrained flow and heat transfer of an electrically conducting nonNewtonian fluid due to a stretching surface subject to partial slip. The constitutive equation of the non-Newtonian fluid is modeled by that for a third grade fluid. They found out that slip decreases the momentum boundary layer thickness and increases the thermal boundary layer thickness, whereas the third grade fluid parameter has an opposite effect on the thermal and velocity boundary layers.

Many efforts were made to find analytical and numerical solutions, imposing certain status and using various mathematical concepts to the same problem [13-19]. The mathematical technique employed in the current analysis is the two-parameter group transformation, which leads to a similarity representation of the problem. Morgan [20] presented a theory which led to amendments over earlier similarity methods and Michal [21] extended Morgan's theory. Group methods, as a class of methods which assuage to a reduction of the number of independent variables, were first presented in [22, 23]. Moran and Gaggioli [24, 25] indicated a general systematic group formalism for similarity study, where a given combination of partial differential equations was reduced to a system of ordinary differential equations [25-34].

In the present work, we provide analytical solution for the unsteady free convection non-Newtonian fluids flow over a continuous moving vertical plate subjected to constant heat flux using a two-parameter group. The specified technique is explained at the following parts. Under the employment of a two-parameter group, the governing partial differential equations and boundary conditions are reduced to ordinary differential equations with the appropriate boundary conditions. The obtained differential equations are solved using the shooting method.

\section{Mathematical Formulation}

The unsteady laminar flow of an incompressible fluid induced by a ceaseless moving sheet placed in a fluid at quiescent is considered. The vertical flat sheet illustrated in Figure 1 arises from a thin slit at $x=y=0$ and is subsequently stretched vertically. The positive $x$ and $y$ coordinates are measured along the direction of the moving film with the slot as the origin and the normal to the sheet, respectively. Constant heat flux, $q_{w}$, is imposed to the flat sheet, giving rise to a

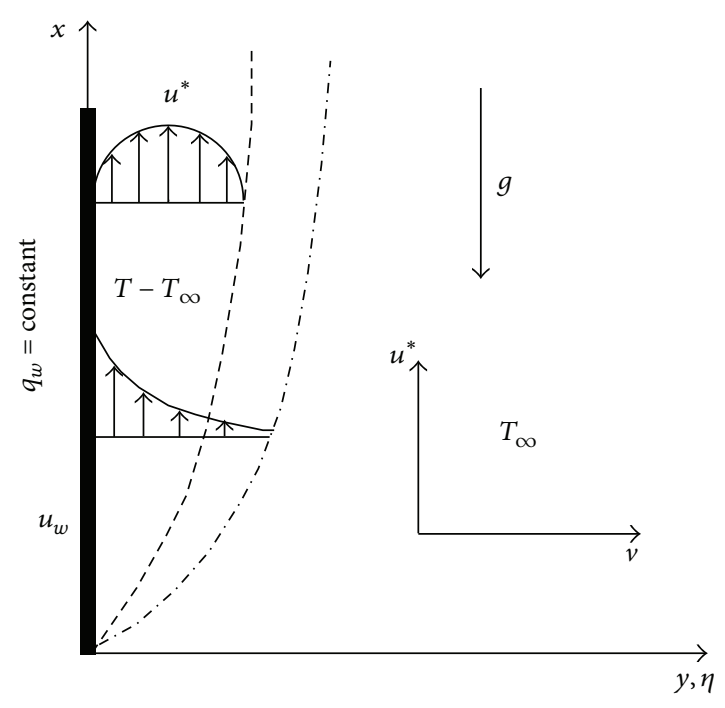

Figure 1: Physical model for laminar flow over stretching sheet.

buoyancy force, while the ambient fluid is kept at a constant temperature, $T_{\mathrm{\infty}}$. The boundary layer equations governing the free convection flow over the moving sheet are expressed as follows:

$$
\begin{gathered}
\frac{\partial u^{*}}{\partial x}+\frac{\partial v}{\partial y}=0 \\
\frac{\partial u^{*}}{\partial t}+u^{*} \frac{\partial u^{*}}{\partial x}+v \frac{\partial u^{*}}{\partial y}=\vartheta n\left(\frac{\partial u^{*}}{\partial y}\right)^{n-1} \frac{\partial^{2} u}{\partial y^{2}}+g \beta\left(T-T_{\infty}\right), \\
\frac{\partial T}{\partial t}+u^{*} \frac{\partial T}{\partial x}+v \frac{\partial T}{\partial y}=\frac{\vartheta}{\operatorname{Pr}} \frac{\partial^{2} T}{\partial y^{2}}
\end{gathered}
$$

where the certain boundary conditions are

$$
\begin{aligned}
& u^{*}(x, y, 0)=u_{0}^{*}(x, y), \quad v(x, y, 0)=v_{0}(x, y), \\
& T(x, y, 0)=T_{0}(x, y), \quad u^{*}(x, 0, t)=u_{w}(x, t), \quad \\
& v(x, 0, t)=0 . \\
& \frac{\partial T(x, 0, t)}{\partial y}=\frac{-q_{w}}{k}, \quad u^{*}(x, y, t)=0, T(x, y, t)=T_{\infty} \\
& \text { as } y \longrightarrow+\infty .
\end{aligned}
$$

$u^{*}$ and $v$ are velocity components related to the $x$ and $y$ coordinates. $T, g$, and $n$ are fluid temperature, gravity, and power index of non-Newtonian fluid, respectively. $\mathrm{Pr}$ is defined as the ratio of kinematic viscosity $(v)$ to thermal diffusion $(\alpha) . \beta$ is thermal expansion coefficient at constant pressure where $u_{0}^{*}, v_{0}$, and $T_{0}$ are initial velocity components and initial temperature. $u_{w}$ and $T_{w}$ are fluid velocity and temperature on vertical moving sheet. Dimensionless $x$ velocity and temperature are defined using the following 
relationships: $u=u^{*} / u_{w}$ and $\theta(x, y, t)=((T(x, y, t)-$ $\left.\left.T_{\infty}\right) / q_{w} x\right) k \sqrt{\operatorname{Re}_{x}}$. Now, (1) is rewritten in the form

$$
\begin{gathered}
u \frac{\partial u_{w}}{\partial x}+u_{w} \frac{\partial u}{\partial x}+\frac{\partial v}{\partial y}=0, \\
u \frac{\partial u_{w}}{\partial t}+u_{w} \frac{\partial u}{\partial t}+u_{w}^{2} u \frac{\partial u}{\partial x}+u^{2} u_{w} \frac{\partial u_{w}}{\partial x}+v u_{w} \frac{\partial u}{\partial y} \\
=\vartheta n u_{w}^{n}\left(\frac{\partial u}{\partial y}\right)^{n-1}\left(\frac{\partial^{2} u}{\partial y^{2}}\right)+\frac{g \beta q_{w}}{k \sqrt{\operatorname{Re}_{x}}} \theta x, \\
x \frac{\partial \theta}{\partial t}+u_{w} u\left(x \frac{\partial \theta}{\partial x}+\theta\right)+v \frac{\partial \theta}{\partial y} x=\frac{\vartheta}{\operatorname{Pr}} x \frac{\partial^{2} \theta}{\partial y^{2}},
\end{gathered}
$$

and boundary conditions are

$$
\begin{aligned}
& u(x, y, 0)=u_{0}(x, y), \quad v(x, y, 0)=v_{0}(x, y), \\
& \theta(x, y, 0)=\theta_{0}(x, y), \quad u(x, 0, t)=1, \quad v(x, 0, t)=0, \\
& \frac{\partial \theta(x, 0, t)}{\partial y}=-\frac{\sqrt{\mathrm{Re}_{x}}}{x}, \quad u(x, y, t)=0, \quad \theta(x, y, t)=0 \\
& \text { as } y \longrightarrow+\infty \text {. }
\end{aligned}
$$

\section{Group Formulation of the Problem}

3.1. Group Formulation. The problem is solved by employing a two-parameter group transformation to the partial differential equations of (3) to (5). This transformation decreases the three independent variables $(x ; y ; t)$ to one similarity variable; $\eta(x ; y ; t)$ and the governing equations of (3) to (5) are transformed to a system of ordinary differential equations in terms of the similarity variable $\eta$. This technique is based on a class of transformation, namely, $G$, including two parameters $\left(a_{1}, a_{2}\right)$ :

$$
G: \bar{S}=C^{S}\left(a_{1}, a_{2}\right) S+K^{S}\left(a_{1}, a_{2}\right),
$$

where $S$ is representative for $x, y, t, u_{w}, v$, and $\theta . C^{s}$ and $K^{s}$ are real valued and at least differentiable in their real arguments $\left(a_{1}, a_{2}\right)$. Dependent variables and related differentiates are as follows via chain rule operations:

$$
\begin{array}{r}
\bar{S}_{\bar{i}}=\left(\frac{C^{S}}{C^{i}}\right) S_{i}, \quad \bar{S}_{\overline{i j}}=\left(\frac{C^{S}}{C^{i} C^{j}}\right) S_{i j} \\
i=x, y, t, \quad j=x, y, t .
\end{array}
$$

For instance, (3) is transformed as follows:

$$
\bar{u} \frac{\partial \bar{u}_{w}}{\partial \bar{x}}+\bar{u}_{w} \frac{\partial \bar{u}}{\partial \bar{x}}+\frac{\partial \bar{v}}{\partial \bar{y}}=H_{1}\left(a_{1}, a_{2}\right)\left[u \frac{\partial u_{w}}{\partial x}+u_{w} \frac{\partial u}{\partial x}+\frac{\partial v}{\partial y}\right],
$$

where $H_{1}\left(a_{1}, a_{2}\right)$ is constant. If (7) and (8) are put in (9),

$$
\begin{aligned}
& {\left[\frac{C^{u} C^{u_{w}}}{C^{x}}\right] u \frac{\partial u_{w}}{\partial x}+\left[\frac{C^{u} C^{u_{w}}}{C^{x}}\right] u_{w} \frac{\partial u}{\partial x}+\left[\frac{C^{v}}{C^{y}}\right] \frac{\partial v}{\partial y}+R_{1}} \\
& =H_{1}\left(a_{1}, a_{2}\right)\left[u \frac{\partial u_{w}}{\partial x}+u_{w} \frac{\partial u}{\partial x}+\frac{\partial v}{\partial y}\right]
\end{aligned}
$$

and $R_{1}$ is $\left[C^{u_{w}} K^{u} / C^{x}\right]\left(\partial u_{w} / \partial x\right)+\left[C^{u} K^{u_{w}} / C^{x}\right](\partial u / \partial x)$. For invariant transformation, $R_{1}$ is equated to zero. This is satisfied by setting

$$
K^{u}=K^{u_{w}}=0 .
$$

Finally, we get

$$
\left[\frac{C^{u} C^{u_{w}}}{C^{x}}\right]=\left[\frac{C^{v}}{C^{y}}\right]=H_{1}\left(a_{1}, a_{2}\right) .
$$

Simultaneously, after, (4) and (5) transformation:

$$
K^{u}=K^{v}=K^{u_{w}}=K^{\theta}=K^{x}=K^{y}=0,
$$

$$
\begin{aligned}
{\left[\frac{C^{u} C^{u_{w}}}{C^{t}}\right] } & =\left[\frac{\left(C^{u}\right)^{2}\left(C^{u_{w}}\right)^{2}}{C^{x}}\right]=\left[\frac{C^{u} C^{v} C^{u_{w}}}{C^{y}}\right] \\
& =\frac{\left(C^{u_{w}}\right)^{n}\left(C^{u}\right)^{n}}{\left(C^{y}\right)^{n+1}}=C^{\theta} C^{x}=H_{2}\left(a_{1}, a_{2}\right),
\end{aligned}
$$

$$
\begin{aligned}
{\left[\frac{C^{\theta} C^{x}}{C^{t}}\right] } & =\left[C^{u} C^{u_{w}} C^{\theta}\right]=\left[\frac{C^{x} C^{v} C^{\theta}}{C^{y}}\right]=\left[\frac{C^{x} C^{\theta}}{\left(C^{y}\right)^{2}}\right] \\
& =H_{3}\left(a_{1}, a_{2}\right)
\end{aligned}
$$

and we have

$$
K^{u}=K^{v}=K^{u_{w}}=K^{\theta}=0 .
$$

Employing invariant transformation for boundary conditions results in

$$
\begin{gathered}
K^{t}=K^{y}=0 \\
C^{u}=1 .
\end{gathered}
$$

Invoking (12) to (15) and (17) reduces to

$$
\begin{gathered}
C^{x}=C^{y}, \quad C^{t}=\left(C^{y}\right)^{2 /(2-n)}, \\
C^{u_{w}}=C^{v}=\frac{1}{\left(C^{y}\right)^{n /(2-n)}}, \quad C^{\theta}=\frac{1}{\left(C^{y}\right)^{4 /(2-n)} .}
\end{gathered}
$$

Group $G$ is summarized as follows:

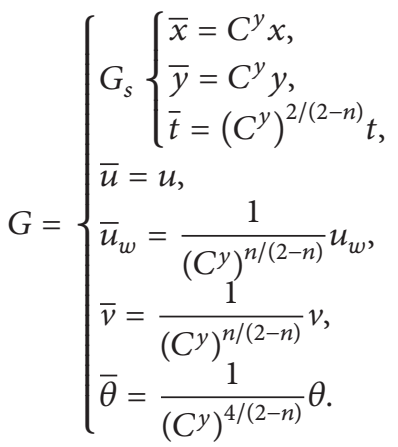


This group converts invariantly the differential equations of (3) to (5) and the initial and boundary conditions (6a) and (6b).

3.2. The Complete Set of Absolute Invariants. The complete set of absolute invariants is as follows:

(i) the absolute invariants of the independent variables $(x, y, t)$ are $\eta=\eta(x, y, t)$

(ii) the absolute invariants of the dependent variables $\left(u, v, u_{w}, \theta\right)$ are

$g_{j}\left(x, y, t: u, u_{w}, v, \theta\right)=F_{j}(\eta(x, y, t)), \quad j=1,2,3,4$.

The basic theorem in group theory asserts that a function $g_{j}\left(x, y, t: u, u_{w}, v, \theta\right)$ is an absolute invariant of a twoparameter group if it satisfies the following two first-order linear differential equations:

$$
\sum_{i=1}^{13}\left(\alpha_{i} S_{i}+\alpha_{i+1}\right) \frac{\partial g}{\partial S_{i}}=0, \quad \sum_{i=1}^{13}\left(\beta_{i} S_{i}+\beta_{i+1}\right) \frac{\partial g}{\partial S_{i}}=0
$$

where $\alpha_{1}=\left(\partial C^{x} / \partial a_{1}\right)\left(a_{1}^{0}, a_{2}^{0}\right), \alpha_{2}=\left(\partial K^{x} / \partial a_{1}\right)\left(a_{1}^{0}, a_{2}^{0}\right)$, $\beta_{1}=\left(\partial C^{x} / \partial a_{2}\right)\left(a_{1}^{0}, a_{2}^{0}\right), \beta_{2}=\left(\partial K^{x} / \partial a_{2}\right)\left(a_{1}^{0}, a_{2}^{0}\right)$, and so forth; $\left(a_{1}^{0}, a_{2}^{0}\right)$ are the identity elements of the group.

The absolute invariant $\eta(x, y, t)$ of the independent variables $(x, y, t)$ is determined using $(21)$ :

$$
\begin{aligned}
& \left(\alpha_{1} x+\alpha_{2}\right) \frac{\partial \eta}{\partial x}+\alpha_{3} y \frac{\partial \eta}{\partial y}+\alpha_{5} t \frac{\partial \eta}{\partial t}=0 \\
& \left(\beta_{1} x+\beta_{2}\right) \frac{\partial \eta}{\partial x}+\beta_{3} y \frac{\partial \eta}{\partial y}+\beta_{5} t \frac{\partial \eta}{\partial t}=0
\end{aligned}
$$

where $\alpha_{4}=\beta_{4}=K^{y}=0$ and $\alpha_{6}=\beta_{6}=K^{t}=0$.

Elimination of $y(\partial \eta / \partial y)$ and $\partial \eta / \partial x$ from (22) yields

$$
\begin{gathered}
\left(\lambda_{31} x+\lambda_{32}\right) \frac{\partial \eta}{\partial x}+\lambda_{35} t \frac{\partial \eta}{\partial t}=0 \\
\left(\lambda_{31} x+\lambda_{32}\right) y \frac{\partial \eta}{\partial y}+\left(\lambda_{15} x+\lambda_{25}\right) t \frac{\partial \eta}{\partial t}=0
\end{gathered}
$$

where $\lambda_{i j}=\alpha_{i} \beta_{j}-\alpha_{j} \beta_{i}$ and $i, j=1,2,3,4,5$. Invoking (21) and definitions of $\alpha$ and $\beta$ we get $\alpha_{5}=2 \alpha_{3}$ and $\beta_{5}=2 \beta_{3}$; thus $\lambda_{35}=\alpha_{3} \beta_{5}-\alpha_{5} \beta_{3}$ will assuage to zero. Using (23a) we obtain $\partial \eta / \partial x=0$ indicating that $\eta$ is dependent on $y$ and $t$. Solving (23b), we get

$$
\eta=y \pi(t)
$$

where $\pi(t)=a t^{b}$ and $a$ and $b$ are arbitrary constants.

Simultaneously, the absolute invariants of the dependent variables $u, v, u_{w}$, and $\theta$ are obtained from the group transformation (19):

$$
\begin{aligned}
& g_{1}(x, y, t ; u)=u(\eta), \\
& g_{2}(x, y, t ; u)=\theta(\eta) .
\end{aligned}
$$

$g_{3}\left(x, t ; u_{w}\right)$ is obtained using $(21)$ as

$$
\begin{aligned}
& \left(\alpha_{1} x+\alpha_{2}\right) \frac{\partial g_{3}}{\partial x}+\left(\alpha_{5} t+\alpha_{6}\right) \frac{\partial g_{3}}{\partial t}+\left(\alpha_{11} u_{w}+\alpha_{12}\right) \frac{\partial g_{3}}{\partial u_{w}}=0 \\
& \left(\beta_{1} x+\beta_{2}\right) \frac{\partial g_{3}}{\partial x}+\left(\beta_{5} t+\beta_{6}\right) \frac{\partial g_{3}}{\partial t}+\left(\beta_{11} u_{w}+\beta_{12}\right) \frac{\partial g_{3}}{\partial u_{w}}=0
\end{aligned}
$$

Eliminating terms of $\partial g_{3} / \partial x$ and $\partial g_{3} / \partial t$ from $((26 a)$ and (26b)) results in

$$
g_{3}\left(x, t ; u_{w}\right)=\phi_{1}\left(\frac{u_{w}}{\omega(x, t)}\right)=E(\eta) .
$$

Similarly, for $g_{4}(x, t ; v)$,

$$
g_{4}(x, t ; v)=\phi_{2}\left(\frac{v}{\Gamma(x, t)}\right)=F(\eta)
$$

where $\omega(x, t), \Gamma(x, t), E(\eta)$, and $F(\eta)$ are functions to be determined. Without loss of generality, the $\phi$ 's in (27) and (28) are selected to be identity functions; hence the functions $u_{w}(x, t)$ and $v(x, y, t)$ are expressed in terms $E(\eta)$ and $F(\eta)$ as

$$
\begin{aligned}
& u_{w}(x, t)=\omega(x, t) E(\eta), \\
& v(x, y, t)=\Gamma(x, t) F(\eta) .
\end{aligned}
$$

Since $\omega(x, t)$ and $u_{w}(x, t)$ are independent of $y$, whereas $\eta$ is function of $y$, we conclude that $E(\eta)$ is equal to a constant such as $E_{0}$. Equation (29) yields

$$
u_{w}(x, t)=E_{0} \omega(x, t)
$$

Without loss of generality $E_{0}$ is equated to one. The functions of $\Gamma(x, t)$ and $\omega(x, t)$ will be determined later on, such that the governing equations of (3) to (5) reduce to a set of ordinary differential equations in $E(\eta), F(\eta)$, and $u(\eta)$.

\section{Reduction to a Set of Ordinary Problem}

Now we define $\eta$ in general form of $\eta=y \pi(x, t)$. Invoking (30) and (31) and (3) to (5),

$$
\begin{gathered}
\frac{d F}{d \eta}+C_{1} \eta \frac{d u}{d \eta}+C_{2} u=0, \\
n\left(\frac{d u}{d \eta}\right)^{n-1} \frac{d^{2} u}{d \eta^{2}}-\left[\eta\left(C_{3}+C_{4} u\right)+C_{5} F\right] \frac{d u}{d \eta} \\
-C_{6} u-C_{7} u^{2}+C_{8} \theta=0, \\
\frac{d^{2} \theta}{d \eta^{2}}-\operatorname{Pr}\left[\frac{d \theta}{d \eta}\left\{\eta\left(C_{10}+C_{11} u\right)+C_{12} F\right\}-C_{9} u \theta\right]=0 .
\end{gathered}
$$


Constants of $C_{1}$ to $C_{12}$ are defined as follows:

$$
\begin{aligned}
& C_{1}=\frac{\omega}{\Gamma \pi^{2}} \frac{\partial \pi}{\partial x} \\
& C_{2}=\frac{1}{\Gamma \pi} \frac{\partial \omega}{\partial x} \\
& C_{3}=\frac{1}{\vartheta \omega^{n-1} \pi^{n+2}} \frac{\partial \pi}{\partial t} \\
& C_{4}=\frac{1}{\vartheta \omega^{n-2} \pi^{n+2}} \frac{\partial \pi}{\partial x} \\
& C_{5}=\frac{\Gamma}{\vartheta \omega^{n-1} \pi^{n}} \\
& C_{6}=\frac{1}{\vartheta \omega^{n} \pi^{n+1}} \frac{\partial \omega}{\partial t} \\
& C_{7}=\frac{1}{\vartheta \omega^{n-1} \pi^{n+1}} \frac{\partial \omega}{\partial x} \\
& C_{8}=\frac{x}{\vartheta \omega^{n} \pi^{n+1}} \frac{g \beta q_{w}}{k \sqrt{\mathrm{Re}_{x}}} \\
& C_{9}=\frac{\omega}{9 x \pi^{2}} \\
& C_{10}=\frac{1}{9 \pi^{3}} \frac{\partial \pi}{\partial t} \\
& C_{11}=\frac{\omega}{9 \pi^{3}} \frac{\partial \pi}{\partial x} \\
& C_{12}=\frac{\Gamma}{9 \pi} \text {. }
\end{aligned}
$$

Now we obtain the exact value of constants as

$$
\frac{C_{7}}{C_{8}}=1 \longrightarrow \omega(x)=\left(\frac{g \beta q_{w}}{k \sqrt{\mathrm{Re}_{x}}}\right)^{1 / 2} x .
$$

From (31),

$$
u_{w}(x)=\left(\frac{g \beta q_{w}}{k \sqrt{\mathrm{Re}_{x}}}\right)^{1 / 2} x
$$

For $C_{9}=1$, we have

$$
\pi=\frac{1}{\vartheta^{1 / 2}}\left(\frac{g \beta q_{w}}{k \sqrt{\mathrm{Re}_{x}}}\right)^{1 / 4} .
$$

According to the definition of similarity variable,

$$
\eta=y\left(\frac{g \beta q_{w}}{\vartheta^{2} k \sqrt{\mathrm{Re}_{x}}}\right)^{1 / 4} .
$$

For $C_{2}=1$, we get

$$
\Gamma=\vartheta^{1 / 2}\left(\frac{g \beta q_{w}}{k \sqrt{\mathrm{Re}_{x}}}\right)^{1 / 4} .
$$

Using (30), the horizontal component of velocity is

$$
v(\eta)=\vartheta^{1 / 2}\left(\frac{g \beta q_{w}}{k \sqrt{\mathrm{Re}_{x}}}\right)^{1 / 4} F(\eta) .
$$

As $\pi$ is invariant and $\omega$ is independent of time,

$$
C_{1}=C_{3}=C_{4}=C_{6}=C_{10}=C_{11}=0 .
$$

On the other hand, due to definitions of $\pi, \omega$, and $\Gamma$,

$$
C_{12}=1, \quad C_{5}=C_{7}=C_{8}=(\omega \pi)^{1-n} .
$$

Substituting the above constants in (32), they finally reduce to

$$
\begin{gathered}
\frac{d F}{d \eta}+u=0, \\
n(\omega \pi)^{n-1}\left(\frac{d u}{d \eta}\right)^{n-1} \frac{d^{2} u}{d \eta^{2}}-F \frac{d u}{d \eta}-u^{2}+\theta=0, \\
\frac{d^{2} \theta}{d \eta^{2}}-\operatorname{Pr}\left(\frac{d \theta}{d \eta} F+u \theta\right)=0 .
\end{gathered}
$$

The new forms of boundary conditions are

$$
\begin{gathered}
F(0)=0, \\
u(0)=1, \quad u(\infty)=0, \\
\frac{\partial \theta(0)}{\partial \eta}=-1, \quad \theta(\infty)=0 .
\end{gathered}
$$

It can be proved that $\partial \theta(0) / \partial \eta=-1$. According to $(2 \mathrm{~b})$ and definition of $\theta,(2 b)$ will change into

$$
\frac{\partial \theta(x, 0, t)}{\partial y}=\frac{-\sqrt{\mathrm{Re}_{x}}}{x},
$$

where $\operatorname{Re}_{x}=u_{s} x / \mathcal{Y}$ and $u_{s}$ is average velocity in $y$ direction. Assuming $u_{s}=u_{w}$,

$$
\operatorname{Re}_{x}=x^{2} \pi^{2}
$$

Using chain rule operation,

$$
\frac{\partial \theta(0)}{\partial \eta} \frac{\partial \eta}{\partial y}=\frac{-\sqrt{\mathrm{Re}_{x}}}{x} .
$$

So, according to $\operatorname{Re}_{x}$ and $\eta, \partial \theta(0) / \partial \eta$ will be -1 .

For instance, assuming $n=1$ for a case of Newtonian fluid flow, (43) will reduce to

$$
\frac{d^{2} u}{d \eta^{2}}+\frac{\eta}{2} \frac{d u}{d \eta}-u=-\operatorname{erfc}\left(\frac{\sqrt{\operatorname{Pr}}}{2} \eta\right)
$$

where

$$
u(0)=1, \quad u(\infty)=0 .
$$


TABLE 1: Comparison of present problem solution and case of Kassem [34] for Pr = 1, 2, 5, and 10 of Newtonian fluid.

\begin{tabular}{|c|c|c|c|c|c|}
\hline \multirow{2}{*}{$\operatorname{Pr}$} & \multicolumn{3}{|c|}{ Kassem [34] } & \multicolumn{2}{|c|}{ Present work } \\
\hline & $\eta$ & $u(\eta)$ & $\Theta(\eta)$ & $u(\eta)$ & $\Theta(\eta)$ \\
\hline \multirow{4}{*}{1} & 0.5 & 0.669 & 0.507 & 0.667 & 0.510 \\
\hline & 1 & 0.391 & 0.256 & 0.381 & 0.261 \\
\hline & 1.5 & 0.165 & 0.103 & 0.161 & 0.105 \\
\hline & 2 & 0.000 & 0.003 & 0.003 & 0.005 \\
\hline \multirow{4}{*}{2} & 0.3 & 0.736 & 0.398 & 0.742 & 0.401 \\
\hline & 0.6 & 0.515 & 0.222 & 0.520 & 0.225 \\
\hline & 0.9 & 0.315 & 0.092 & 0.317 & 0.095 \\
\hline & 1.2 & 0.143 & 0.009 & 0.150 & 0.011 \\
\hline \multirow{4}{*}{5} & 0.25 & 0.745 & 0.206 & 0.750 & 0.208 \\
\hline & 0.5 & 0.525 & 0.095 & 0.531 & 0.011 \\
\hline & 0.75 & 0.333 & 0.040 & 0.335 & 0.042 \\
\hline & 1 & 0.171 & 0.017 & 0.171 & 0.017 \\
\hline \multirow{4}{*}{10} & 0.2 & 0.768 & 0.124 & 0.771 & 0.125 \\
\hline & 0.4 & 0.565 & 0.046 & 0.571 & 0.048 \\
\hline & 0.6 & 0.381 & 0.015 & 0.391 & 0.017 \\
\hline & 0.8 & 0.216 & 0.007 & 0.218 & 0.008 \\
\hline
\end{tabular}

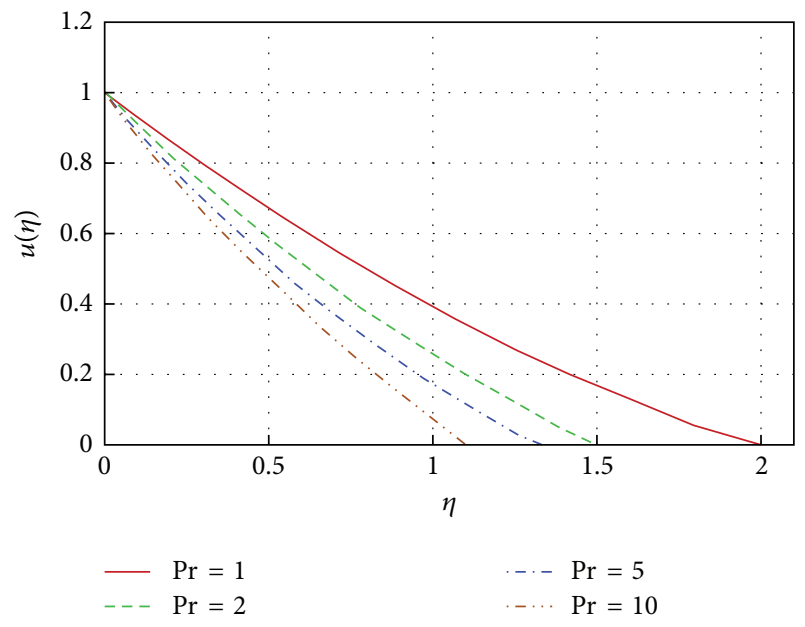

Figure 2: Vertical velocity of Newtonian fluid at the vicinity of stretching sheet for different values of Pr.

\section{Results and Discussion}

In this section, initially, results of Newtonian fluid flow over stretching sheet are compared with Kassem [34]. Table 1 shows the results obtained by numerical solution of ODE equation, (49), and [34] for power index, $n$ equal to unity and Pr number of 1 . There is a satisfying agreement among the present evaluations. Complementary results for different values of $\mathrm{Pr}$ are shown in Figures 2 and 3. It can be understood that, as the fluid momentum diffusivity increases with respect to the thermal diffusivity, large Pr numbers, vertical velocity decreases. In other words, the velocity $u(\eta)$ inside the boundary layer decreases with the increase of fluid viscosity. Also, the temperature plot of boundary layer reveals a decrease of temperature at the wall temperature for larger

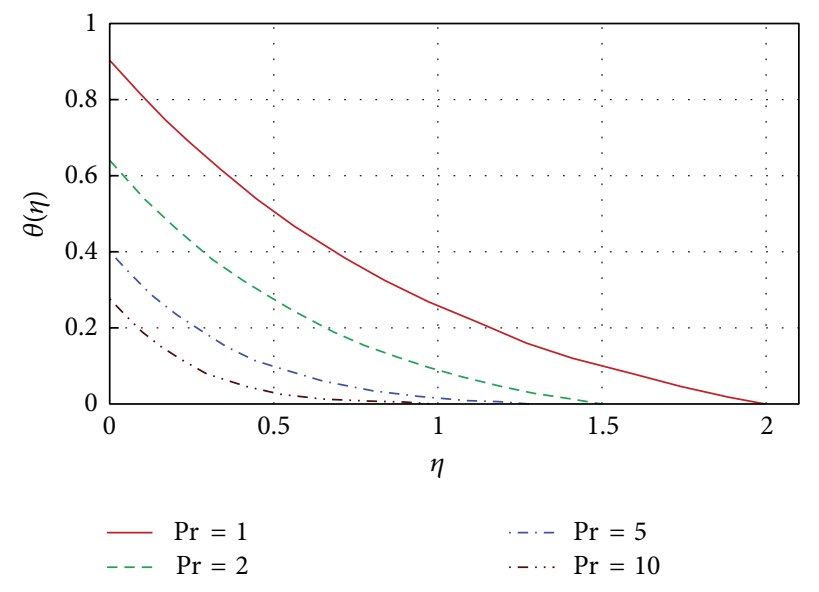

FIgURE 3: Temperature of Newtonian fluid at the vicinity of stretching sheet for different values of Pr.

Pr. So heat loss increases for larger Pr as the boundary layer gets thinner.

For non-Newtonian case of the fluid, the vertical velocity and temperature of fluid adjacent to the stretching sheet are evaluated by solving (42) to (44) with the certain boundary conditions of (45). Results of the shooting method solution are illustrated in Figures 4 and 5 for $\operatorname{Pr}=5$. As the power index increases, vertical velocity component encounters with resistance to rise due to increased value of shear stress. But, for lower power index, vertical component of velocity moves faster due to reduced apparent viscosity. Similarly, thinner thermal boundary layer for higher power index will increase the amount of heat loss. 


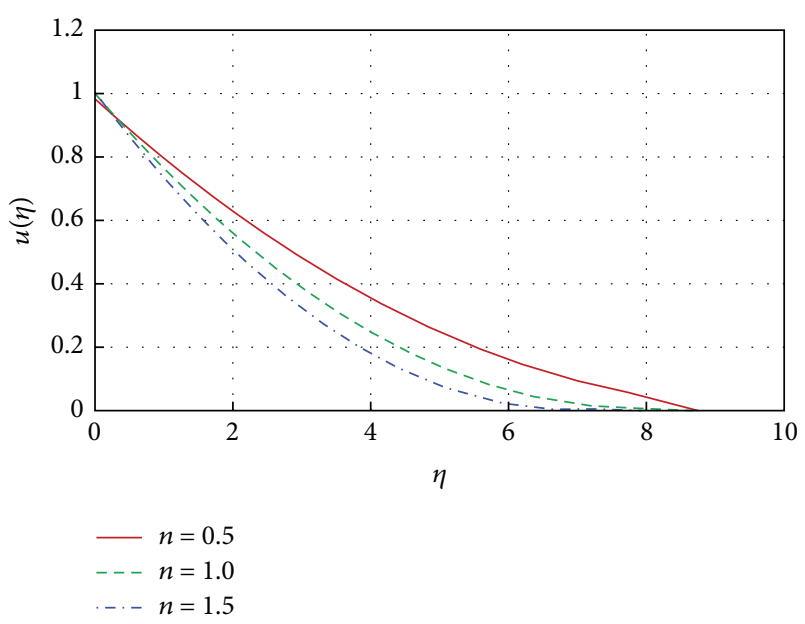

FIGURE 4: Vertical velocity of non-Newtonian fluid at the vicinity of stretching sheet for constant values of $\operatorname{Pr}=5$.

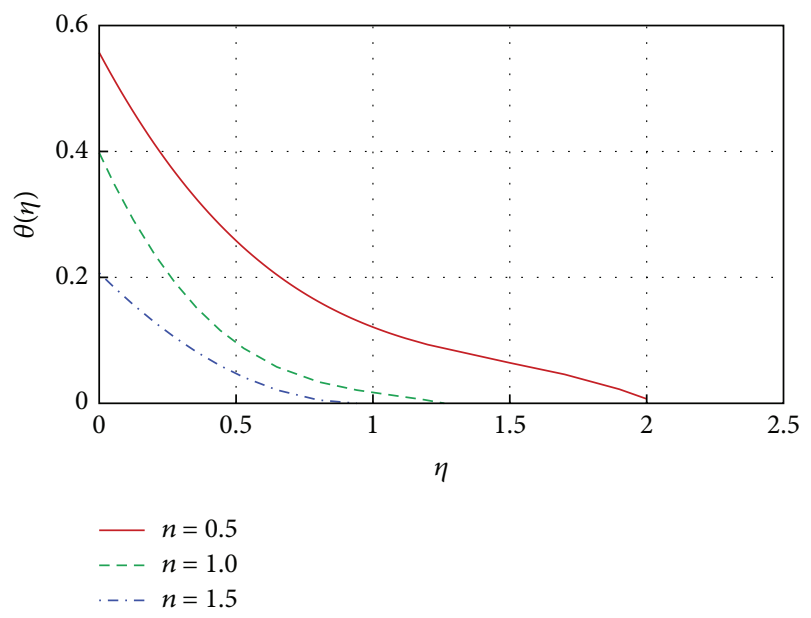

FIgURE 5: Temperature of non-Newtonian fluid at the vicinity of stretching sheet for constant values of $\operatorname{Pr}=5$.

\section{Conclusion}

Similarity solutions are performed for flow of power law non-Newtonian fluid film on unsteady stretching surface subjected to constant heat flux. The nonlinear coupled partial differential equations (PDE) governing the flow and the boundary conditions are transformed to a set of ordinary differential equations (ODE) using two-parameter groups. This technique reduces the number of independent variables by two, and conclusively the obtained ordinary differential equations are solved numerically for the temperature and velocity using the shooting method. Results show that higher Pr number and higher power index of non-Newtonian fluid encounter with difficulty to move as faster as lower Pr and power index. Enhanced amount of shear stress explains the reason of the predicted flow.

\section{Conflict of Interests}

The authors declare that there is no conflict of interests regarding the publication of this paper.

\section{References}

[1] C. Y. Wang, "Liquid film on an unsteady stretching surface," Quarterly of Applied Mathematics, vol. 48, no. 4, pp. 601-610, 1990.

[2] H. I. Andersson, J. B. Aarseth, and B. S. Dandapat, "Heat transfer in a liquid film on an unsteady stretching surface," International Journal of Heat and Mass Transfer, vol. 43, no. 1, pp. 69-74, 2000.

[3] R. Usha and R. Sridharan, "The axisymmetric motion of a liquid film on an unsteady stretching surface," Journal of Fluids Engineering, vol. 117, no. 1, pp. 81-85, 1995.

[4] B. S. Dandapat, B. Santra, and H. I. Andersson, "Thermocapillarity in a liquid film on an unsteady stretching surface," International Journal of Heat and Mass Transfer, vol. 46, no. 16, pp. 3009-3015, 2003.

[5] H. I. Andersson and T. Ytrehus, "Falkner-Skan solution for gravity-driven film flow," Journal of Applied Mechanics, vol. 52, no. 4, pp. 783-786, 1985.

[6] H. I. Andersson and F. Irgens, "Gravity-driven laminar film flow of power-law fluids along vertical walls," Journal of NonNewtonian Fluid Mechanics, vol. 27, no. 2, pp. 153-172, 1988.

[7] D.-Y. Shang and H. I. Andersson, "Heat transfer in gravitydriven film flow of power-law fluids," International Journal of Heat and Mass Transfer, vol. 42, no. 11, pp. 2085-2099, 1998.

[8] C.-H. Chen, "Heat transfer in a power-law fluid film over a unsteady stretching sheet," Heat and Mass Transfer, vol. 39, no. 8-9, pp. 791-796, 2003.

[9] K. Vajravelu and A. Hadjinicolaou, "Heat transfer in a viscous fluid over a stretching sheet with viscous dissipation and internal heat generation," International Communications in Heat and Mass Transfer, vol. 20, no. 3, pp. 417-430, 1993.

[10] A. Mehmood and A. Ali, "Analytic solution of generalized three-dimensional flow and heat transfer over a stretching plane wall," International Communications in Heat and Mass Transfer, vol. 33, no. 10, pp. 1243-1252, 2006.

[11] R. Tsai, K. H. Huang, and J. S. Huang, "Flow and heat transfer over an unsteady stretching surface with non-uniform heat source," International Communications in Heat and Mass Transfer, vol. 35, no. 10, pp. 1340-1343, 2008.

[12] B. Sahoo and Y. Do, "Effects of slip on sheet-driven flow and heat transfer of a third grade fluid past a stretching sheet," International Communications in Heat and Mass Transfer, vol. 37, no. 8, pp. 1064-1071, 2010.

[13] C. R. Illingworth, "Unsteady laminar flow of gas near an infinite flat plate," Mathematical Proceedings of the Cambridge Philosophical Society, vol. 46, no. 4, pp. 603-613, 1950.

[14] R. Siegel, "Transient free convection from a vertical flat plate," Transactions of the ASME, vol. 80, no. 2, pp. 347-359.

[15] B. Gebhart, "Transient natural convection from vertical elements," Journal of Heat Transfer, vol. 83, pp. 61-70, 1961.

[16] A. J. Chamkha, "Similarity solution for thermal boundary layer on a stretched surface of a non-Newtonian fluid," International Communications in Heat and Mass Transfer, vol. 24, no. 5, pp. 643-652, 1997.

[17] J. D. Hellums and S. W. Churchill, "Transient and steady state, free and natural convection, numerical solutions: part I-the 
isothermal, vertical plate," AIChE Journal, vol. 8, no. 5, pp. 690692, 1962.

[18] G. D. Callahan and W. J. Marner, “Transient free convection with mass transfer on an isothermal vertical flat plate," International Journal of Heat and Mass Transfer, vol. 19, no. 2, pp. 165-174, 1976.

[19] T. G. Howell, D. R. Jeng, and K. J. de Witt, "Momentum and heat transfer on a continuous moving surface in a power law fluid," International Journal of Heat and Mass Transfer, vol. 40, no. 8, pp. 1853-1861, 1997.

[20] A. J. A. Morgan, "The reduction by one of the number of independent variables in some systems of partial differential equations," The Quarterly Journal of Mathematics, vol. 3, no. 1, pp. 250-259, 1952.

[21] A. D. Michal, "Differential invariants and invariant partial differential equations under continuous transformation groups in normed linear spaces," Proceedings of the National Academy of Sciences of the United States of America, vol. 37, pp. 623-627, 1951.

[22] G. Birkhoff, "Mathematics for engineers," Electrical Engineering, vol. 67, no. 12, pp. 1185-1188, 1948.

[23] G. Birkhoff, Hydrodynamics: A Study in Logic, Fact and Similitude, Princeton University Press, Princeton, NJ, USA, 1960.

[24] M. J. Moran and R. A. Gaggioli, "Reduction of the number of variables in systems of partial differential equations, with auxiliary conditions," SIAM Journal on Applied Mathematics, vol. 16, no. 1, pp. 202-215, 1968.

[25] M. J. Moran and R. A. Gaggioli, "A new systematic formalism for similarity analysis, with application to boundary layer flows," Technical Summary Report 918, U.S. Army Mathematics Research Center, 1968.

[26] W. F. Ames, "Similarity for the nonlinear diffusion equation," Industrial \& Engineering Chemistry Fundamentals, vol. 4, no. 1, pp. 72-76, 1965.

[27] W. F. Ames, Nonlinear Partial Differential Equations in Engineering, chapter 2, Academic Press, New York, NY, USA, 1972.

[28] W. F. Ames and M. C. Nucci, "Analysis of fluid equations by group methods," Journal of Engineering Mathematics, vol. 20, no. 2, pp. 181-187, 1986.

[29] M. J. Moran and R. A. Gaggioli, "Similarity analysis of compressible boundary layer flows via group theory," Technical Summary Report 838, U.S. Army Mathematics Research Center, Madison, Wis, USA, 1967.

[30] M. J. Moran and R. A. Gaggioli, "Similarity analysis via group theory," AIAA Journal, vol. 6, pp. 2014-2016, 1968.

[31] M. J. Moran and R. A. Gaggioli, "A new systematic formalism for similarity analysis, with application to boundary layer flows," Technical Summary Report 918, U.S. Army Mathematics Research Center, 1968.

[32] M. J. Moran and R. A. Gaggioli, "Reduction of the number of variables in systems of partial differential equations, with auxiliary conditions," SIAM Journal on Applied Mathematics, vol. 16, no. 1, pp. 202-215, 1968.

[33] M. B. Abd-el-Malek, M. M. Kassem, and M. L. Mekky, "Similarity solutions for unsteady free-convection flow from a continuous moving vertical surface," Journal of Computational and Applied Mathematics, vol. 164, no. 1, pp. 11-24, 2004.

[34] M. Kassem, "Group solution for unsteady free-convection flow from a vertical moving plate subjected to constant heat flux," Journal of Computational and Applied Mathematics, vol. 187, no. 1, pp. 72-86, 2006. 


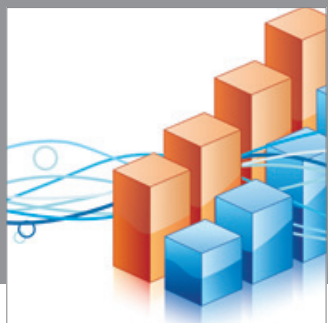

Advances in

Operations Research

mansans

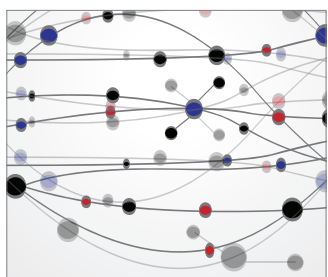

The Scientific World Journal
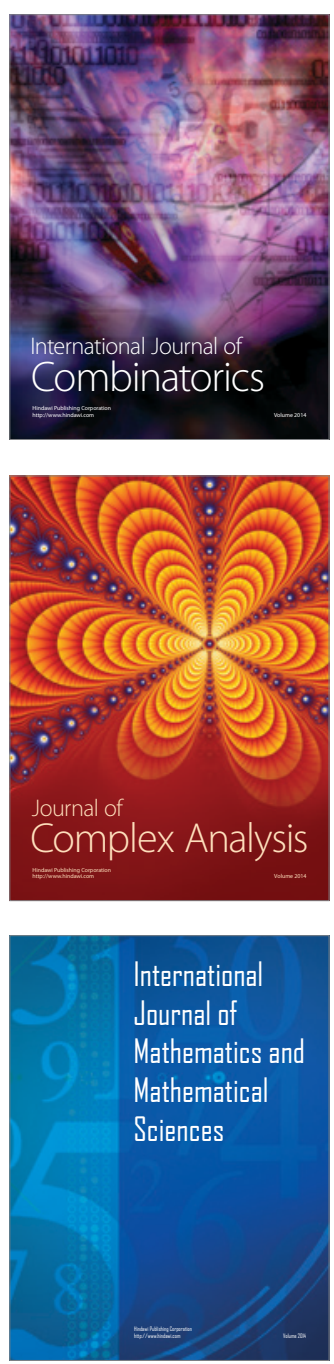
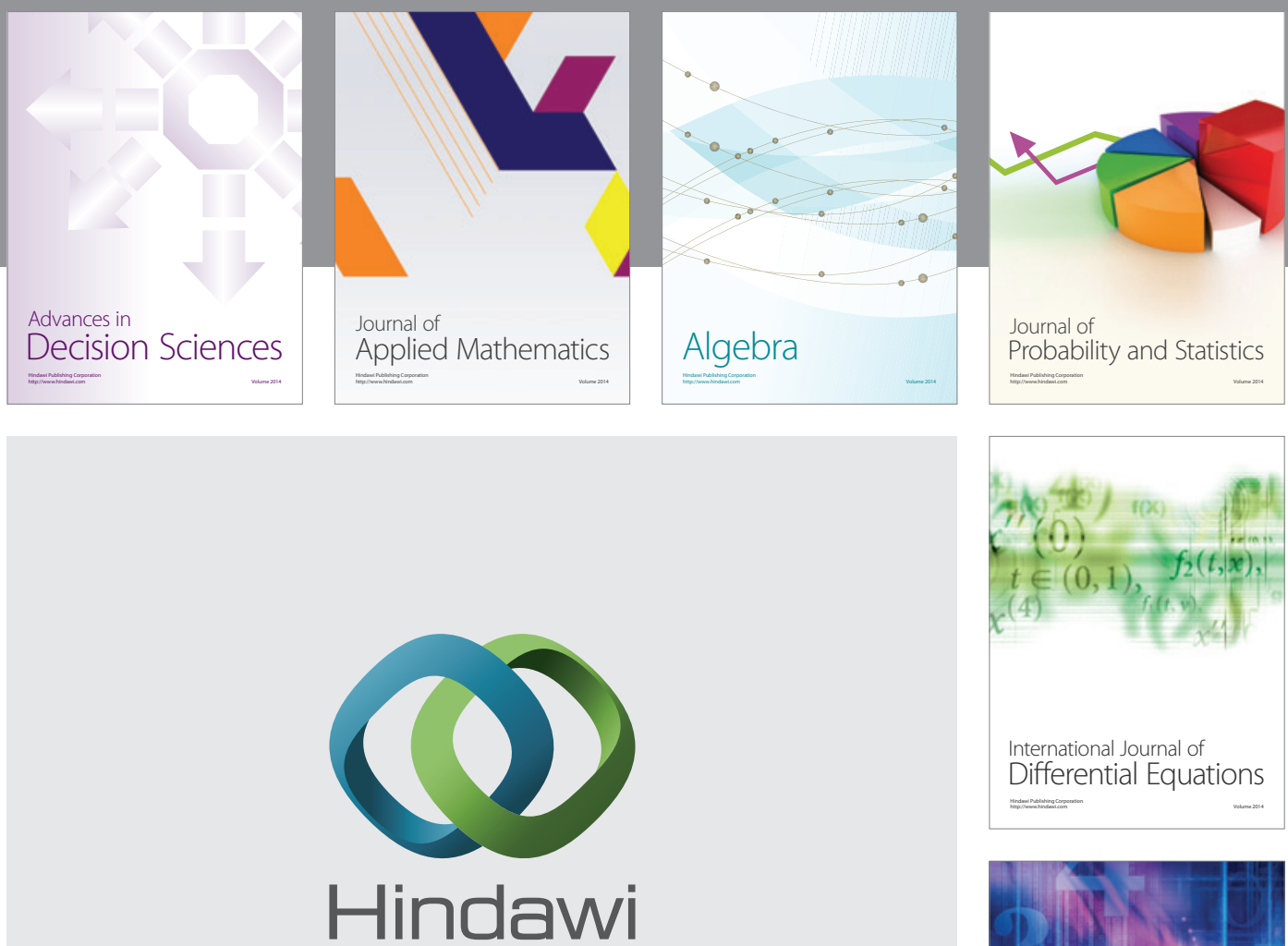

Submit your manuscripts at http://www.hindawi.com
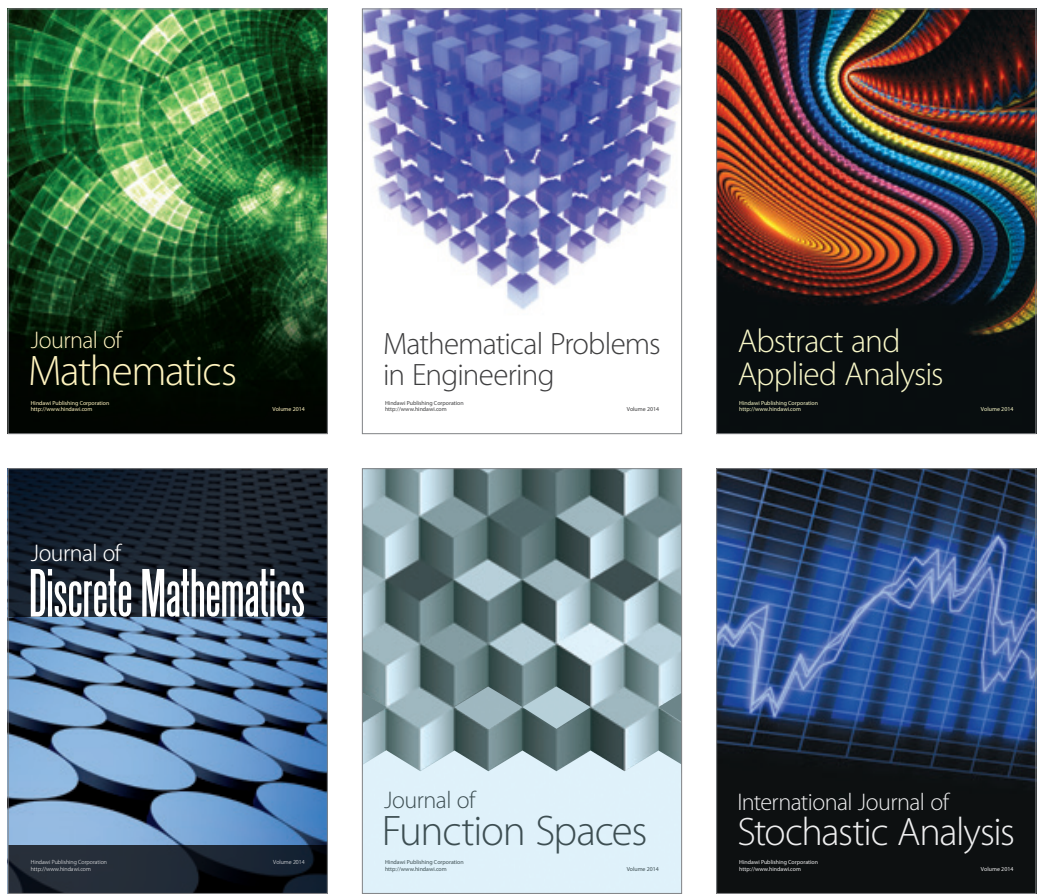

Journal of

Function Spaces

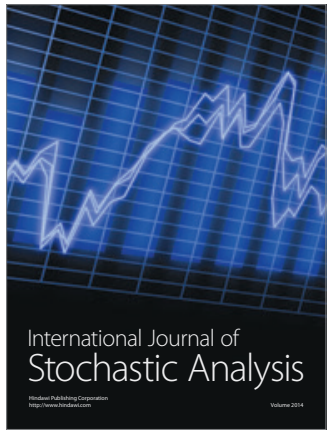

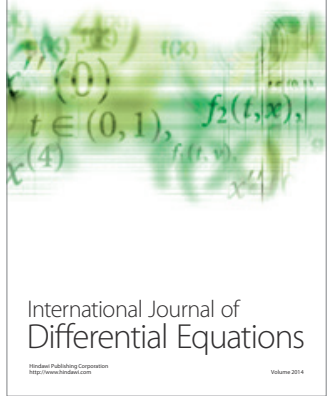
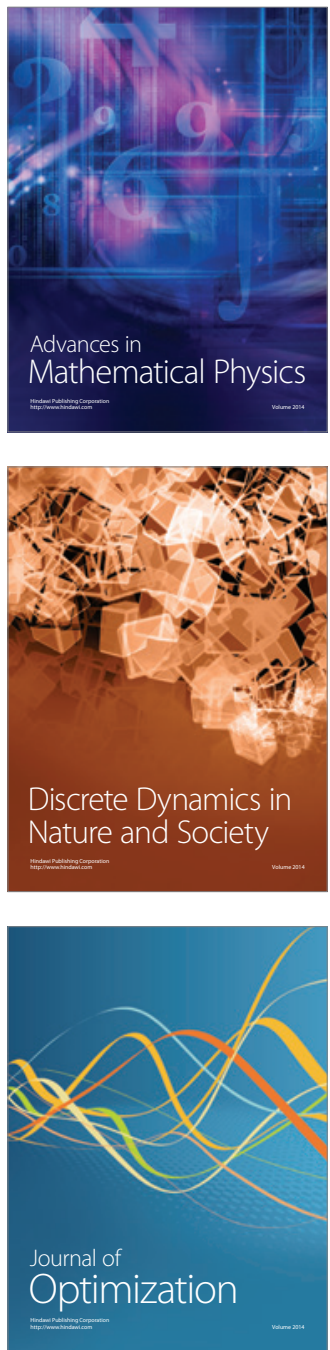